\title{
Intervista a Maurizio Gallieni
}

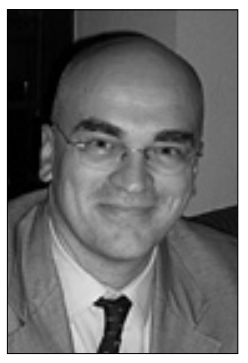

Maurizio Gallieni, MD

UO Nefrologia e Dialisi

AO Ospedale San Carlo Borromeo

Milano

gallieni.maurizio@sancarlo.mi.it

Nel centro ove presti attività qual è la percentuale di pazienti monitorati per CKD-MBD stadio 3-5 e quali marker biologici usi a tal fine?

Tutti i pazienti con clearance della creatinina $<60 \mathrm{ml} /$ mn (ovvero stadi CKD 3, 4 e 5) vengono monitorati per l'insorgenza di alterazioni del metabolismo minerale. In stadio 5 è pratica comune richiedere, oltre a calcemia e fosforemia, anche i livelli di PTH, fosfatasi alcalina e, più recentemente, di $25(\mathrm{OH})$ vitD. Tuttavia, va ricordato che nello stadio 3 e in molti pazienti in stadio 4 raramente si osservano alterazioni dei livelli di calcemia e fosforemia, Per questo motivo, anche se non tutti i nefrologi sono sensibilizzati al problema, sarebbe importante dosare anche in questi pazienti i livelli di PTH e di $25(\mathrm{OH})$ vitD per identificare precocemente deficit di vitamina $\mathrm{D}$ e lo sviluppo di iperparatiroidismo secondario nelle fasi precoci, quando è agevolmente controllabile con la terapia medica. La frequenza dei controlli ematici è variabile, secondo lo stadio di malattia ed il riscontro di alterazioni degli esami. In stadio 5, il dosaggio di PTH viene effettuato ogni 3-6 mesi, la 25(OH)vitD ogni 6-12 mesi.

Nel centro ove presti attività pratichi il dosaggio della $25(\mathrm{OH}) \mathrm{D}$ e/o della $1,25(\mathrm{OH})_{2} \mathrm{D} 3$ ?

Se si, in quale tipo di paziente ed in quale percentuale? Se no, perché ritieni di non doverla praticare?

Il dosaggio di 25(OH)vitD è un indice affidabile delle scorte di vitamina $\mathrm{D}$, anche se non è ancora stato stabilito quale sia il livello ideale di $25(\mathrm{OH})$ vitD (1). Per molto tempo non è stato preso in considerazione, in quanto si riteneva che nel paziente con insufficienza re- nale l'anomalia fondamentale del sistema fosse la ridotta attività della 1-alfa-idrossilasi renale e quindi il deficit di calcitriolo. Sembrava quindi inutile aumentare il livello di substrato - ovvero di $25(\mathrm{OH})$ vitD - di un enzima inattivo, quando era possibile somministrare direttamente la molecola carente, ovvero il calcitriolo. Tuttavia, negli ultimi anni è emerso che sia nella popolazione generale che nei pazienti nefropatici il deficit di $25(\mathrm{OH})$ vitD si associa ad aumento della morbilità e mortalità. Inoltre, è noto che anche cellule extrarenali (ad esempio i macrofagi) esprimono 1-alfa-idrossilasi, con un'attività paracrina del sistema ormonale vitamina $\mathrm{D}$ indipendente dal deficit enzimatico renale (2). Per questo motivo molti centri, compreso il nostro, hanno iniziato a dosare la $25(\mathrm{OH})$ vitD per valutare e correggere il deficit di $25(\mathrm{OH})$ vitD, come indicato dalle recenti linee guida KDIGO (3). Non dosiamo routinariamente la $1,25(\mathrm{OH})_{2}$ vitD, perché la decisione sul trattamento con calcitriolo si basa ancora sui livelli di PTH. Negli stadi avanzati di CKD moltissimi pazienti hanno valori ridotti di $1,25(\mathrm{OH})_{2}$ vitD, ma ancora nessuno ha stabilito se ci sia comunque un vantaggio a normalizzarli. Il dosaggio di $1,25(\mathrm{OH})_{2}$ vitD è utile nella diagnosi differenziale dell'ipercalcemia con bassi valori di PTH.

Applichi la correzione di 25(OH)D in caso di carenza? Perché, con quale protocollo ed in quale tipo di paziente?

Ritengo che in questa fase della ricerca clinica su CKDMBD, che ha evidenziato il problema del grave deficit di $25(\mathrm{OH})$ vitD nella maggioranza dei pazienti nefropatici, esiste un'opportunità unica, di randomizzare i pazienti a correzione del deficit vs placebo (o non trattamento), valutando gli effetti della terapia su morbilità e mortalità. La modalità di correzione che utilizzo attualmente si basa sulla somministrazione di calcifediolo $-25(\mathrm{OH})$ vitD - (10 gtt/die per una o due confezioni di prodotto), seguito da un mantenimento con colecalciferolo (20 gtt/ settimana $=5000$ Unità/settimana).

Nel centro ove presti attività in quale percentuale o in quali casi clinici particolari pratichi la biopsia ossea?

La biopsia ossea viene praticata molto raramente, dato che la disponibilità di diversi esami ematochimici riesce 
a darci un’idea abbastanza affidabile dello stato metabolico dell'osso. Tuttavia, credo che sia sotto-utilizzata, in quanto è ormai chiaro che esiste un range di valori di PTH alquanto ampio (dai 100 ai $500 \mathrm{pg} / \mathrm{ml}$ ) in cui non possiamo sapere con certezza se il rimaneggiamento osseo è basso, normale o elevato. $\mathrm{Ci}$ sono poi i casi di dolori ossei e di fratture patologiche, che in molti casi potrebbero essere meglio chiariti con la biopsia. I due principali ostacoli all'esecuzione della biopsia ossea sono l'invasività della manovra e la complessità della lettura istologica. Per superarli, credo che le soluzioni siano: 1. eseguire la biopsia in sedazione (come ormai si verifica routinariamente per le colonscopie); 2 . centralizzare la lettura della biopsia in pochi centri qualificati.

Nel centro ove presti attività in quale percentuale ed in quali stadi di CKD usi indagini strumentali per la ricerca delle calcificazioni vascolari? Quali indagini utilizzi?

La ricerca delle calcificazioni vascolari viene effettuata routinariamente allinizio del trattamento dialitico con radiografia laterale della colonna vertebrale lombare (calcificazioni aortiche) e con ecocardiografia, valutando le calcificazioni valvolari. Nei pazienti con stadi più precoci di CKD, questa valutazione viene effettuata in caso di alterazioni dei parametri del metabolismo minerale, su base individuale.

Nel centro ove presti attività quale è la percentuale di pazienti in trattamento sostitutivo che resta nel range di normalità (secondo le linee guida SIN) per Ca, P e PTH?

Calcio 50\%, Fosforo 66\%, PTH 32\%.

Nel centro ove presti attività quale è la percentuale dei vari chelanti usati per ricercare il controllo del Ca-P nei pazienti in trattamento sostitutivo (anche in off-label treatment)?

Il 62\% dei pazienti in dialisi extracorporea usa sali di calcio, il $32 \%$ sevelamer, il 10\% carbonato di lantanio, il 6\% sali di alluminio. Tranne che nei casi di ipercalcemia, i chelanti di ultima generazione vengono associati a basse dosi di sali di calcio.

Nel centro ove presti attività quale è la percentuale di pazienti in CKD 3-5 sottoposta a dieta ipoproteica per il controllo della iperfosforemia?

A tutti i pazienti viene consigliata una riduzione dell'introito di proteine animali, senza alimenti aproteici. A pazienti motivati e selezionati, circa il 10\%, viene prescritta una dieta con alimenti aproteici.
Nel centro ove presti attività quale percentuale di pazienti in trattamento sostitutivo assume vit-D od analoghi, calciomimetico, od entrambi?

Calcitriolo per os $62 \%$; calcitriolo o analoghi e.v. $12 \%$, calciomimetico $6 \%$.

Le calcificazioni vascolari -evento davvero comune nel paziente con CKD- sono associate ad un elevato rischio di mortalità in dialisi ma non esistono studi d'intervento che dimostrino chiaramente che la loro riduzione migliori la sopravvivenza. Come va interpretato tale apparente paradosso?

Molti studi randomizzati fatti su pazienti dializzati hanno dato risultati negativi e per certi versi sconfortanti. Tra i più importanti vanno ricordati lo studio 4D (4) e lo studio Aurora (5) (effetto statine sulla riduzione della colesterolemia e sulla mortalità), gli studi sugli agenti stimolanti l'eritropoiesi (6) e, più specificatamente nell'ambito della CKD-MBD, lo studio DCOR sull'effetto di sevelamer vs sali di calcio sulla mortalità (7). Lo studio DCOR non ha valutato l'effetto della terapia sulle calcificazioni vascolari, per cui non è possibile dire che un intervento terapeutico che riduca la progressione delle calcificazioni vascolari sia inutile. Più semplicemente non è stato ancora provato, con uno studio adeguatamente dimensionato, che controllare le calcificazioni migliori la morbilità e la mortalità, come ci si aspetterebbe in base agli studi osservazionali. Lo studio Treat to Goal (8) ha chiaramente dimostrato che è possibile rallentare la progressione delle calcificazioni con una terapia chelante con sevelamer, privo di calcio e con effetti pleiotropici positivi (riduzione della colesterolemia, riduzione dello stato infiammatorio). Lo studio RIND (9) ha dimostrato una riduzione significativa della mortalità nei pazienti trattati con Sevelamer rispetto a pazienti trattati con solo calcio. Questo studio su pazienti incidenti in dialisi ha dimostrato anche che i pazienti con calcificazioni vascolari hanno una mortalità superiore ai pazienti senza calcificazioni. Va sottolineato che il periodo di follow-up dello studio RIND è stato di 5 anni, e che la differenza tra i due gruppi di pazienti si evidenziava dopo il terzo anno di osservazione. Lo studio DCOR ha dimostrato una riduzione significativa della mortalità (assumendo che come nello studio Treat to Goal il sevelamer rallentasse la progressione delle calcificazioni) solo in sottogruppi: nei pazienti con più di 65 anni (perché presumibilmente questa categoria di pazienti, più fragile, ha avuto un numero superiore di eventi che ha consentito di raggiungere una significatività statistica); nei pazienti trattati per almeno 2 anni (in seguito ad un'analisi post-hoc, pubbli- 
cata solo in forma di abstract). Il processo che porta allo sviluppo di calcificazioni e quindi all'aumento del rischio di morte probabilmente richiede, sulla base dei risultati disponibili, almeno 3-5 anni. In sintesi, le calcificazioni vascolari restano seriamente indiziate di colpevolezza, ma per dimostrare il loro ruolo nella genesi di complicanze importanti (morbilità e mortalità cardiovascolare) sarebbero necessari studi di grandi dimensioni (simili al DCOR, che aveva arruolato 2000 pazienti), con un follow-up di almeno 5 anni, possibilmente includendo solo pazienti che presentano già calcificazioni all'inizio dello studio, perché pazienti privi di calcificazioni molto difficilmente sviluppano calcificazioni ed eventi clinici ad esse correlate in soli 5 anni.

L'iperfosforemia è oramai considerata al pari di altri fattori di rischio noti, come un 'killer' per il paziente uremico. Esistono evidenze sulla capacità di questo elemento nell'indurre meccanismi di variazione fenotipica a livello della cellula muscolare liscia verso cellule capaci di creare matrice ossea e quindi deposizione di fosfato di calcio. $\grave{E}$ il solo fosfato capace di tutto ciò o sono implicati altri mediatori in questo processo? Quale ruolo gioca/no nel processo di ossificazione vascolare? Quali altre novità ci possiamo attendere dalla ricerca in un prossimo futuro?

Il processo di calcificazione vascolare è molto più complesso di quanto potevamo immaginare solo 10 anni fa (10). Per molti anni si è pensato che la calcificazione fosse un fenomeno aspecifico legato al superamento di determinati livelli di calcio e di fosforo, sostanzialmente una precipitazione passiva per sovra-saturazione di questi due elementi. Ora è stato chiarito che nel processo di calcificazione operano fattori che favoriscono la precipitazione di calcio e fosforo in modo organizzato (ossificazione dei vasi, con formazione di idrossiapatite organizzata in lamelle) e fattori che inibiscono le calcificazioni, come la fetuina (circolante), l'osteopontina e la Matrix Gla Protein (MGP, tissutale). Importantissimi sono i segnali che agiscono sulle cellule vascolari, determinandone la trasformazione fenotipica in cellule di tipo osteoblastico o modulando la sintesi di fattori che favoriscono la mineralizzazione (ad esempio Cbfa1). In alcuni casi, la stessa molecola può avere effetti diversi secondo la sua concentrazione: ad esempio è noto che in caso di intossicazione da vitamina $\mathrm{D}$ si osserva ipercalcemia e calcificazioni, ma a dosi fisiologiche il calcitriolo può avere un'azione di inibizione delle calcificazioni. La segnalazione che i topi knock-out per FGF23 e per Klotho sviluppano estese calcificazioni cardiovascolari e dei tessuti molli, ha aperto un ampio fronte di ricerca che sta chiarendo il ruolo di queste due molecole nella pato- genesi dell'iperfosfatemia del paziente con insufficienza renale e dello sviluppo di calcificazioni (11).

È pensabile che ci ritroveremo a curare la proteinuria con una 'semplice' vitamina (la D od $i$ suoi analoghi)?

Gli studi sperimentali hanno in effetti dimostrato un ruolo del sistema ormonale vitamina $\mathrm{D}$ nel controllo della proteinuria (12). Difficilmente vedremo un controllo di tutte le molteplici cause di nefropatia proteinurica da parte della vitamina $\mathrm{D}$, che potrà invece avere un ruolo adiuvante nei casi non adeguatamente controllati con altri farmaci che contrastano il sistema renina-angiotensina. Attendiamo l'esito di studi clinici randomizzati, tra cui lo studio VITAL già completato (13), per avere conferma dell'efficacia antiproteinurica del sistema vitamina D nell'uomo.

Perché supplementare con calcifediolo e calcitriolo $i$ pazienti in CKD 3-5, con carenza di 25(OH)D?

Le considerazioni di base per rispondere a questa domanda sono le stesse esposte nella risposta alla domanda sul dosaggio dei livelli di $25(\mathrm{OH})$ vitD e $1,25(\mathrm{OH})_{2}$ vitD. Non è possibile formulare una risposta esauriente, supportata da dati derivati da studi randomizzati e controllati. La conoscenza della fisiopatologia del sistema vitamina $D$ e i risultati di dati sperimentali ed osservazionali indicano in modo coerente che entrambe le molecole sono carenti nei pazienti con CKD. Supplementare i pazienti solo con $25(\mathrm{OH})$ vitD, in una condizione di scarsa attività dell'enzima 1-alfa-idrossilasi renale, potrebbe non correggere adeguatamente il deficit di calcitriolo. D'altra parte supplementare i pazienti solo con calcitriolo, la molecola più attiva sul VDR, potrebbe non essere sufficiente per normalizzare le alterazioni legate all'attività di 1-alfa-idrossilai tissutali.

\section{Commento conclusivo}

La comprensione della fisiologia e fisiopatologia umana è un processo senza fine. Capire di più ci apre nuovi orizzonti, anche terapeutici, ma spesso comporta anche l'apertura di nuove domande e ci rendiamo conto che meccanismi che credevamo semplici sono molto più complessi, con interazioni tra molecole e tipi cellulari molteplici. Il campo della CKD-MBD, una volta oggetto dell'attenzione di pochi appassionati, si è espanso enormemente ad ha fatto grandi progressi. Sono state sviluppate nuove terapie, al punto che il nefrologo clinico a volte è indeciso, per la complessità dell'argomento, su 
come utilizzarle in modo combinato, efficiente ed economico.

Un ulteriore sviluppo che auspico è la maggiore comprensione dei sistemi di bilancio corporeo di calcio e fosforo. L'organismo sano regola molto finemente questi bilanci, secondo le necessità di crescita e di mantenimento dell'attività metabolica dell'organismo. Nel paziente con insufficienza renale, in cui i meccanismi che mantengono questo bilancio si alterano, siamo abituati a prendere le nostre decisioni terapeutiche basandoci sui livelli ematici di calcio, fosforo e PTH. In futuro sarebbe molto utile poter invece valutare l'andamento dei bilanci corporei di calcio e di fosforo, per integrarli con gli altri dati che già abbiamo a disposizione e quindi per prendere decisioni terapeutiche più efficaci, volte a prevenire e/o rallentare la progressione delle calcificazioni vascolari.

\section{Bibliografia}

1. Bischoff-Ferrari HA, Giovannucci E, Willett WC, Dietrich T, Dawson-Hughes B. Estimation of optimal serum concentrations of 25-hydroxyvitamin D for multiple health outcomes. Am J Clin Nutr. 2006; 84: 18-28.

2. Gallieni M, Kamimura S, Ahmed A, Bravo E, Delmez J, Slatopolsky E, Dusso A. Kinetics of monocyte 1 alpha-hydroxylase in renal failure. Am J Physiol. 1995; 268 : F746-53.

3. Kidney Disease: Improving Global Outcomes (KDIGO) CKD-MBD Work Group. KDIGO clinical practice guideline for the diagnosis, evaluation, prevention, and treatment of Chronic Kidney Disease-Mineral and Bone Disorder (CKD-MBD). Kidney Int Suppl. 2009;(113):S1-130
4. Wanner C, Krane V, März W, Olschewski M, Mann JF, Ruf G, Ritz E; German Diabetes and Dialysis Study Investigators. Atorvastatin in patients with type 2 diabetes mellitus undergoing hemodialysis. New Engl J Med. 2005; 353: 238-48.

5. Fellström BC, Jardine AG, Schmieder RE, et al. Rosuvastatin and cardiovascular events in patients undergoing hemodialysis. New Engl J Med. 2009; 360: 1395-407.

6. Unger EF, Thompson AM, Blank MJ, Temple R. Erythropoiesis-stimulating agents-time for a reevaluation. $\mathrm{N} \mathrm{Engl}$ J Med. 2010; 362: 189-92.

7. Suki WN, Zabaneh R, Cangiano JL, et al. Effects of sevelamer and calcium-based phosphate binders on mortality in hemodialysis patients. Kidney Int. 2007; 72: 1130-7.

8. Chertow GM, Burke SK, Raggi P; Treat to Goal Working Group. Sevelamer attenuates the progression of coronary and aortic calcification in hemodialysis patients. Kidney Int. 2002; 62: 245-52.

9. Block GA, Raggi P, Bellasi A, Kooienga L, Spiegel DM. Mortality effect of coronary calcification and phosphate binder choice in incident hemodialysis patients. Kidney Int. 2007; 71: 438-41.

10. Brancaccio D, Gallieni M, Pasho S, et al. Pathogenesis and treatment of vascular calcification in CKD. G Ital Nefrol. 2009; 26 (Suppl 45):S20-7.

11. Memon F, El-Abbadi M, Nakatani T, Taguchi T, Lanske B, Razzaque MS. Does FGF23-Klotho activity influence vascular and soft tissue calcification through regulating mineral ion metabolism? FGF23, Klotho, and calcification. Kidney Int 2008; 74: 566-570

12. Agarwal R. Vitamin D, proteinuria, diabetic nephropathy, and progression of CKD. Clin J Am Soc Nephrol 2009; 4: 1523-1528.

13. Lambers Heerspink HJ, Agarwal R, et al.. The selective vitamin $\mathrm{D}$ receptor activator for albuminuria lowering (VITAL) study: study design and baseline characteristics. Am J Nephrol. 2009; 30: 280-6. 